\title{
De Gourcy Constance et Chachoua Kamel, Mobilités et migrations en Méditerranée. Vers une anthropologie de l'absence?
}

\section{Simeng Wang}

\section{(2) OpenEdition Journals}

Édition électronique

URL : https://journals.openedition.org/remi/16227

DOI : $10.4000 /$ remi. 16227

ISSN : $1777-5418$

Éditeur

Université de Poitiers

\section{Édition imprimée}

Date de publication : 30 décembre 2020

Pagination : 358-360

ISBN : 979-10-90426-67-2

ISSN : 0765-0752

Référence électronique

Simeng Wang, « De Gourcy Constance et Chachoua Kamel, Mobilités et migrations en Méditerranée Vers une anthropologie de l'absence? ", Revue européenne des migrations internationales [En ligne], vol. 36 - n² et 3 | 2020, mis en ligne le 01 janvier 2021, consulté le 04 janvier 2023. URL : http:// journals.openedition.org/remi/16227 ; DOI : https://doi.org/10.4000/remi.16227

Ce document a été généré automatiquement le 4 janvier 2023.

Tous droits réservés 


\title{
De Gourcy Constance et Chachoua Kamel, Mobilités et migrations en Méditerranée. Vers une anthropologie de l'absence?
}

\author{
Simeng Wang
}

\section{RÉFÉRENCE}

De Gourcy Constance et Chachoua Kamel (Dirs.) (2018) Mobilités et migrations en Méditerranée. Vers une anthropologie de l'absence ?, Revue des mondes musulmans et de la Méditerranée. - 144. - 320 p., ISBN : 979-1-032-00195-0

1 S'inscrivant dans les perspectives ouvertes par le transnationalisme, ce numéro de revue propose une analyse approfondie sur l'expérience de l'absence, en considérant cette dernière comme n'étant « pas seulement le contraire de la présence [...], mais une institution de sens qui définit un système de places et de modalités relationnelles entre les membres géographiquement éloignés d'un collectif donné »(p.14). Réunissant douze articles structurés en trois parties, ce numéro de revue manifeste trois apports majeurs, de natures conceptuelle, épistémologique et théorique.

Il s'agit d'abord de prolonger les pensées d'Abdelmalek Sayad à propos de l'appréhension des mobilités géographiques avec et à partir des sociétés d'émigration, en raffinant nos connaissances sur les formes multiples - matérialisées, symboliques et métaphoriques - de l'absence. En effet, « être absent signifie maintenir des liens avec les membres du groupe d'appartenance afin de ne pas devenir "étranger" au groupe ou considéré comme "disparu"» (p.14). Maintenir des liens à distance implique inévitablement les recompositions des liens sociaux : de parenté, de couple, d'amitié, de citoyenneté, etc. Kamel Chachoua analyse, à partir d'une archive orale et musicale kabyle des années 1940-1960, le rite de «l'appel » (asiwel) essentiellement féminin qui 
consiste à faire revenir l'émigré égaré (amjah). L'évolution de ce rite dans le temps illustre en partie l'éthos de la société kabyle en mutation : l'émigration n'étant plus un tort, mais souhaité, désiré et recherché par bon nombre de jeunes, les rapports entre l'émigré absent et ses proches qui restent au pays ne sont plus les mêmes. Eckehard Pistrick s'intéresse à la sonorité du vide et à la quête du sens de l'absence par des migrants eux-mêmes et leurs proches. Il met en lumière une ambiguïté émotionnelle et une dynamique processuelle de nostalgie intimement liées à l'expérience migratoire. Eftychia Mylona, quant à elle, étudie la communauté grecque (les Aigyptiotes) installée en Égypte entre 1961 et 1976, en adoptant le concept de "multidirectional memory ». L'analyse montre que les récits des acteurs ordinaires remettent en question l'amnésie historique apparue dans les historiographies nationales et les narratives officielles; et permettent de nouvelles compréhensions de l'histoire des Aigyptiotes où coexistent l'absence et la présence.

3 La dimension processuelle que ce numéro accorde à l'absence constitue son deuxième apport au champ des études migratoires. Ne pas considérer l'absence comme un état et un constat, mais comme une dynamique marquée par une temporalité, une temporalité de cycle de vie, mais également de trajectoire et de mobilité, permet de donner à voir l'expérience temporelle de l'absence, qui est à la fois structurée par l'ensemble des relations sociales à distance et structurante, puisque l'absence recompose également ces dernières. Plusieurs articles du numéro traitent de la question de la mort et des retours post-mortem en migration : Azzedine Kinzi analyse les rapatriements à Tounef (Kabylie) des dépouilles d'immigrés décédés à Saint-Étienne en France; Valérie Cuzol étudie les pratiques funéraires des immigrés algériens, tunisiens et marocains à Chalon-sur-Saône en France; Lisa Anteby-Yemini examine quant à elle les rites funéraires de demandeurs d'asile érythréens (de religion chrétienne) et soudanais (de religion musulmane) en Israël. À travers les rites chargés de mémoire, les devenirs des défunts et les choix d'inhumation entre terre étrangère et terre natale, il s'agit de mettre en lumière, d'un côté, la fabrique de l'absence et de la présence par les politiques étatiques vis-à-vis des ressortissants émigrés ; et de l'autre, la capacité d'agir des acteurs et de leurs proches, afin d'accorder un sens à l'appartenance : l'absence et/ ou la présence sont ainsi désirées et négociées. La dimension processuelle de l'absence est également présente dans la contribution de Laura Odasso, qui étudie les trajectoires des femmes ressortissantes du Maroc et du Liban vivant dans des couples binationaux en France. Elle montre le processus de resocialisation en migration, ainsi que le rôle de ces femmes dans le changement de sociétés, tout en étant elles-mêmes en changement.

Enfin, le troisième apport du numéro spécial réside dans sa façon d'appréhender l'absence depuis les politiques d'État ou depuis la vie politique du pays d'origine. Plusieurs contributions mettent en lumière les cadres juridiques de l'absence, ainsi que les catégories étatiques de l'absence: les conditions de binationalité, les statuts des descendants issus du mariage binational, les conditions de rapatriements, les droits d'inhumation en terre natale, etc. Emmanuel Blanchard, à partir des archives du Service des affaires indigènes nord-africaines (SAINA), analyse les demandes d'intervention adressées dans les années 1930 par les familles kabyles, en vue de la recherche de leurs proches " égarés » en région parisienne. Malika Gouirir s'intéresse à un statut particulier de citoyenneté: «Marocains résidant à l'étranger » (MRE), qui vise, du point de vue étatique, à consolider le lien entre ressortissant et Royaume. Ce lien d'affiliation ne peut en aucun cas être rompu ou oublié. L'auteure met en exergue les vécus de ce statut par les individus concernés sur différentes scènes sociales. 
D'autres articles se focalisent sur les expressions et participations politiques à distance des émigrés à l'égard de la vie politique dans le pays d'origine. Claire Demesmay, Sabine Russ-Sattar et Katrin Sold analysent les manières dont les diasporas tunisiennes en France, Allemagne et Italie ont perçu les soulèvements populaires et le changement de régime dans leur pays d'origine en 2011. Léo Fourn étudie l'engagement militant des activistes syriens depuis leur pays d'exil dans l'objectif de maintenir des liens politiques avec la " révolution syrienne".

Outre ces trois apports, le numéro spécial ouvre deux pistes de réflexion sur la question de l'absence en migrations internationales. La première traite du rôle du numérique et des nouveaux médias, à travers l'analyse des MRE numérique - personnes actives sur les réseaux sociaux globaux - (Malika Gouirir), des espaces de résistance en ligne formés par des Tunisiens de l'Europe (Claire Demesmay, Sabine Russ-Sattar et Katrin Sold), et de l'usage d'Internet et plus particulièrement des réseaux sociaux que les activistes syriens en exil utilisent pour tenter d'atténuer l'absence (Léo Fourn). Si l'usage du numérique et des nouveaux médias dans la vie quotidienne des personnes migrantes n'est pas nouveau, certaines contributions à ce numéro ont exploré les liens et les fossés entre "le monde virtuel/en ligne» et "le monde réel/hors-ligne", remettant en question les sens d'absence et de présence dans le contexte migratoire. La seconde piste de réflexion qui mérite d'être approfondie constitue la dimension subjective de l'absence en migration. Certains articles évoquent les émotions, sentiments et souffrances engendrées à partir de l'absence. La subjectivité des individus vivant une situation d'absence en migration, ainsi que la subjectivation des «absents » seront autant des questions de recherche prometteuses à sonder.

De manière générale, en suivant la perspective des travaux d'Abdelmalek Sayad, ce numéro spécial de la Revue des mondes musulmans et de la Méditerranée invite à renouveler les réflexions sur le transnationalisme, à travers les différentes expériences et formes d'absence en migration dans l'espace méditerranéen. Ayant parfaitement réussi à déconstruire la vision dualiste entre la présence et l'absence, cette publication collective incarne, en plus de ses apports en anthropologie, sociologie, science politique et droit, la pensée philosophique de Tchouang-tseu, à propos du « vide en plénitude ».

\section{AUTEURS}

\section{SIMENG WANG}

Sociologue, Chargée de recherche au CNRS, CERMES3 (CNRS-EHESS-INSERM-Université Paris Descartes) 